\title{
ANTIBIOTIC SUSCEPTIBILITY PROFILE OF SALMONELLA ENTERICA SEROVARS: TREND OVER THREE YEARS SHOWING RE-EMERGENCE OF CHLORAMPHENICOL SENSITIVITY AND RARE SEROVARS
}

Sir,

Typhoid and paratyphoid fevers are of major public health concern due to the emergence of resistance to fluoroquinolones, the presently recommended first line of therapy. Reports on treatment failure after administration of ciprofloxacin to patients with enteric fever are increasing. ${ }^{[1]}$ Among the nontyphoidal Salmonellae, Salmonella enterica serovar Typhimurium has been reported to show multidrug resistance. ${ }^{[2]}$ The study reports the antibiotic susceptibility profile of Salmonella spp., highlighting the re-emergence of chloramphenicol sensitivity and rising resistance to ciprofloxacin, in addition to the isolation of rare serovars of Salmonella enterica.

Salmonella spp. ( $n=124)$ isolated from 3,956 blood samples of suspected enteric fever cases, collected during August 2003 to July 2006, were included in the study. Serological typing of the isolates was done at the National Salmonella and Escherichia Center, Central Research Institute, Kasauli, India. All the isolates were screened for susceptibility to antimicrobial drugs like amoxycillin, cotrimoxazole, chloramphenicol, cefuroxime, ceftriaxone, ciprofloxacin and nalidixic acid by the disk diffusion method as per Clinical 
and Laboratory Standard Institute (CLSI) guidelines. ${ }^{[3]}$ Minimum inhibitory concentration (MIC) of ciprofloxacin and nalidixic acid was determined by the standard broth dilution method according to CLSI (formerly NCCLS)

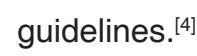

Various Salmonella enterica serovars isolated from blood cultures were $S$. Typhi (71), $S$. Paratyphi A (37), S. Typhimurium (13), S. Welteverdine (01), $S$. Bareilly (01) and $S$. Infantis (01).

All the nontyphoidal Salmonella serovars were isolated from immunocompromised patients. All isolates were sensitive to cefuroxime and ceftriaxone, the drugs of choice for ciprofloxacin treatment failures. Drug resistance pattern of the Salmonella serotypes is shown in Table 1.

Among the 54 ciprofloxacin-sensitive $S$. Typhi isolates, 15 were sensitive and 39 were resistant to nalidixic acid by the disk diffusion method. Fifteen $S$. Typhi isolates that were sensitive to both nalidixic acid and ciprofloxacin by disk diffusion showed an MIC range of $0.062-0.125 \mu \mathrm{g} / \mathrm{ml}$ for ciprofloxacin. Thirtynine isolates that were resistant to nalidixic acid and sensitive to ciprofloxacin had an Ta

Table 1: Resistance pattern and minimum inhibitory concentration of ciprofloxacin and nalidixic acid for $S$ Typhi and S. Paratyphi A isolates

\begin{tabular}{|c|c|c|c|c|c|c|c|c|c|c|}
\hline \multirow[t]{2}{*}{ Year } & \multirow{2}{*}{$\begin{array}{l}\text { Salmonella } \\
\text { serovars }\end{array}$} & \multirow{2}{*}{$\begin{array}{l}\text { No. of } \\
\text { strains }\end{array}$} & \multicolumn{6}{|c|}{ Drug-resistance pattern } & \multicolumn{2}{|c|}{ MIC range $(\mu \mathrm{g} / \mathrm{ml})$} \\
\hline & & & $\overline{A+C}+\mathrm{Co}$ & $A$ & $C$ & Co & $C f$ & $\mathrm{Na}(\%)$ & Cifrofloxacin & $\begin{array}{c}\text { Nalidixic } \\
\text { acid }\end{array}$ \\
\hline 2003-04 & $\begin{array}{c}\text { S. Typhi } \\
\text { Syphos }\end{array}$ & 23 & $05(22 \%)$ & 01 & 03 & - & $\begin{array}{l}011 \\
041\end{array}$ & $\begin{array}{c}12(52) \\
08(100)\end{array}$ & $\begin{array}{l}0.062-0.5 \\
0.062-1.0\end{array}$ & $\begin{array}{l}16-256 \\
32-256\end{array}$ \\
\hline 2004-05 & $\begin{array}{l}\text { S. Paratyphi A } \\
\text { S. Typhi }\end{array}$ & 26 & $02(7 \%)$ & - & - & - & $01 \mathrm{R}, 08 \mathrm{I}$ & $24(92)$ & $0.062-4.0$ & $\begin{array}{l}32-256 \\
16-512\end{array}$ \\
\hline & S. Paratyphi A & 11 & & 01 & 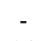 & - & 041 & $10(91)$ & $0.062-1.0$ & $16-512$ \\
\hline 2005-06 & S. Typhi & 22 & $02(9 \%)$ & - & 01 & 01 & 01R, 06I & $20(90)$ & $0.156-4.0$ & $16-512$ \\
\hline & S. Paratyphi A & 18 & & - & & & 041 & $16(89)$ & $0.062-1.0$ & 16-512 \\
\hline
\end{tabular}

$=$ sensitive range, $A=$ Ampic
$I=$ Intermediately susceptible

MIC range of $0.25-1.0 \mu \mathrm{g} / \mathrm{ml}$ for ciprofloxacin. MIC of $0.50-2.5 \mu \mathrm{g} / \mathrm{ml}$ was recorded for 13 $S$. Typhi isolates that were categorized as intermediately susceptible to ciprofloxacin by disk diffusion. Two ciprofloxacin-resistant and two intermediately susceptible isolates of $S$. Typhi had an MIC of $4 \mu \mathrm{g} / \mathrm{ml}$.

Among the $37 \mathrm{~S}$. Paratyphi A strains, 25 were sensitive to ciprofloxacin and 3 were sensitive to nalidixic acid by disk diffusion. The ciprofloxacin MIC of sensitive isolates was $<0.125 \mu \mathrm{g} / \mathrm{ml}$, and the intermediately susceptible isolates $(n=12)$ showed a value between 0.250 and $1.0 \mu \mathrm{g} / \mathrm{ml}$. Eight isolates of $S$. Typhimurium were sensitive, and five were resistant to nalidixic acid by disk diffusion. Among the 'nalidixic acid'-resistant strains, 3 were found to be intermediately susceptible to ciprofloxacin by disk diffusion. MIC of intermediately susceptible strains varied from $0.25 \leq 1 \mu \mathrm{g} / \mathrm{ml}$

तns

The incidence of multidrug resistance (MDR - resistance to three or more antibiotics) in $S$. Typhi strains was $22 \%$ in the first year, $7 \%$ in the second year and $9 \%$ in the third year of the study. MDR was not observed among the isolates of $S$. Paratyphi $A$ and $S$. Typhimurium in the present study.
It is interesting to note that there is a gradua increase in the number of strains of Salmonella resistant to nalidixic acid (MIC $\geq 32 \mu \mathrm{g} / \mathrm{ml}$ ), and these nalidixic acid resistant strains showed reduced susceptibility to ciprofloxacin (MIC $0.25-1.0 \mu \mathrm{g} / \mathrm{ml}$ ). Although the level of reduced susceptibility to ciprofloxacin is below that regarded as clinically significant, an increasing number of treatment failures at this level has been noted. This suggests that current CLSI break point $(4 \mu \mathrm{g} / \mathrm{ml})$ for ciprofloxacin may not accurately predict clinical response to treatment of patients with extra-intestinal salmonellosis.

Two isolates of $S$. Typhi had MIC of $4 \mu \mathrm{g} / \mathrm{ml}$, which is regarded as typical resistance to ciprofloxacin by CLSI criteria. ${ }^{[4]}$ But by disk diffusion, these isolates were found to be intermediately susceptible. These results clearly show that determination of MIC is necessary for every isolate of Salmonella, in order to accurately detect the reduced susceptibility and typical resistance to ciprofloxacin. In the present study, it was found that isolates with reduced susceptibility to ciprofloxacin were resistant to nalidixic acid by disk diffusion. Hence nalidixic acid screening test can be used to detect reduced susceptibility to ciprofloxacin. This will help clinicians to decide on an alternative antibiotic to avoid treatment failures.

2 .

Chloramphenicol sensitivity in S. Typhi (>90\%) has been increasing in different regions of India. ${ }^{[1,5]}$ As noted in the present study, reemergence of sensitivity (94.4\%) and reduced resistance $(5.6 \%)$ to chloramphenicol in $S$. Typhi isolates make it essential to reconsider chloramphenicol as the antibiotic of choice for enteric fever in place of ciprofloxacin or third- generation cephalosporins, the present treatment regimen of typhoid fever. There have been very few reports on the isolation of rare serovars like S. Weltverdine, S. Bareilly, S. Typhimurium and $S$. Infantis from blood cultures.

Due to an increase in the immunocompromise population, these rare serovars of Salmonella enterica, which are susceptible to commonly used antibiotics, might have been isolated. However, even these rare serovars may develop resistance to antibiotics in due course and the policy of empirical treatment for enteric fever and septicemia needs to be rationalized.

\section{ACKNOWLEDGMENT}

The author thanks the National Salmonella and Escherichia Centre, Central Research Institute Kasauli, India, for serotyping the Salmonella isolates.

ic

\section{REFERENCES} 1. Mandal S, Mandal MD, Pal NK. Antibiotic
resistance of Salmonella enterica serovar Paratyphi A in India: Emerging and reemerging problem. J Postgrad Med 2006;52:163-6.

2. Molbak K, Baggesen DL, Arestrup FM, Ebbese JM, Engberg J, Frydendahl K, et al. An outbreak of multidrug resistant, Quinolone resistan Salmonella enterica serotype Typhimurium DT 104. N Engl J Med 1999;341:1420-5.

3. Clinical and Laboratory Standards Institute. Performance standards for antimicrobial disk susceptibility tests; $8^{\text {th }}$ ed. CLSI: 2005. p. M02 A8.

4. National Committee for Clinical Laboratory Standards. Method for dilution antimicrobial susceptibility tests for bacteria that grow 
aerobically. $5^{\text {th }}$ ed. NCCLS Approved standard: 2000. p. M7-A5.

5. Mandal S, Mandal MD, Pal NK. Reduced minimum inhibitory concentration of chloramphenicol for Salmonella enterica serovar Typhi. Indian J Med Sci 2004;58:16-23.
B. DHANASHREE

Department of Microbiology, Kasturba Medical College, Mangalore, India

Correspondence:

Dr. (Mrs.) Dhanashree B., Department of Microbiology, Kasturba Medical College, Mangalore - 575 001, India. E-mail: dbiranthabail@yahoo.co.in 